\title{
Effect of 8 weeks of stretching, strength, and central stability exercises on scoliosis of boy students
}

\author{
Mohammadbagher Forghani Ozrudi@
}

Physical Education Teacher, Education Office, Babol, Iran.

\begin{abstract}
This study was conducted to determine the effect of 8 weeks of stretching, strength, and central stability exercises on scoliosis in boy aged 9 to 13 years. The study includes a comparative model. The statistical population of the present study was boy scoliosis, of whom 60 were scoliosis voluntarily participated in this study \& were randomly divided into three groups of 20 people: Experimental Group 1 (EG 1): Tensile, strength; Experimental Group 2 (EG 2): Tensile, strength training and central stability, Control Group CG). Scoliosis was used to measure scoliosis between the first to the 12th breast (C7 to S1) and the photogrammetric method. The results showed that the mean lateral curvature angle of EG 1 and EG 2 decreased significantly after participating in the correctional exercise program. There was no significant difference between EG 1 and EG 2. The results of the ANOVA test showed that EG 1 and EG 2 were significantly different from CG. The results of this study showed that the corrective exercises used in this study might have an effective effect on reducing the lateral curvature of the spinal column of students with scoliosis.
\end{abstract}

Keywords. Boy Students, central stability, scoliosis, strength, stretching.

\section{Introduction}

The quality and condition of the human body are of particular importance in human life because the resulting positive and negative changes affect other human conditions as well (Forghani Ozrudi \& Nikkhoo Amiri, 2021). The spine plays different roles in the body, one of the most important of which is supporting the chest. The chest area needs a lot of support due to the presence of internal organs, especially the heart, in which the spine plays a major role (Balzini et al., 2003). According to scientific definitions, Scoliosis is a threedimensional deformity of the spine and trunk, including lateral flexion and rotation of the vertebrae with sagittal plane changes (Naqvi, 2019).

Mahaudens et al. (2009) showed that walking length in participants with scoliosis was reduced by $7 \%$ compared to normal participants, and shoulder-pelvic and hip movements in the transverse plane were reduced, and hip movements in the horizontal plane were also reduced.
Naqvi (2019) reported that there was muscle weakness in participants with scoliosis in the lateral muscles of the pelvis-hip and leg. This weakness causes the body to deviate to the sides and even in the anterior-posterior direction. As a result of this, the patients use other muscles to maintain balance.

Richardson et al., based on anatomical, biomechanical, and physiological characteristics, divide muscles into two groups: Stabilizing and moving. The stabilizing muscle group is used to maintain anti-gravity position and function, and the second muscle group is known to create torque in the body (Richarson et al., 1992). It consists of muscles that connect both ends of the muscle or just the Lumbopelvic Hip Complex (LPHC) to the central trunk or lumbar-pelvic-femoral girdle of a muscle to the lumbar spine (Crisco \& Panjabi, 1991).

The muscles of the central trunk are responsible for maintaining the stability of the spine and pelvis and are very important for transferring energy from the trunk to the upper and lower limbs during activities (Tse et

冈 M. Forghani Ozrudi, e-mail: mohammadbagher.forghani@gmail.com

Received: April 18, 2021 - Accepted: June 04, 2021 - Published: June 30, 2021

To Cite: Forghani Ozrudi, M. (2021). Effect of 8 weeks of stretching, strength, and central stability exercises on scoliosis of boy students. Turk J Kinesiol, 7(2), 59-65. DOI: 10.31459/turkjkin.919399 
al., 2005). The similarity of the central area of the trunk is the stable foundation of the trunk (Zazulak et al., 2007).

The most important stabilizing muscles of the spine are the muscles that straighten the spine, whose bilateral action causes the trunk to open, and their unilateral action causes the spine to bend laterally. The internal and external oblique muscles of the abdomen and the lumbar quadriceps are also affected by scoliosis and are short on one side (Sadd et al., 2009). The main muscles of the central stability region or lumbar-pelvic-thigh complex include the spine straightener, abdominal transverse, multi-headed, internal oblique, diaphragm, and pelvic floor muscles (Schmidt \& Lee, 2011; Bergmark, 1989). Richardson \& Jull (1995) hypothesized that optimal spinal stability required contraction of the transverse abdominal muscle and the multicellular muscle.

Simultaneous contraction of the transverse abdominal muscles and the pectoralis major muscle can lead to the retraining of the local (deep) stabilizing muscles (Ghiasi et al, 2007); Defects or inefficiencies in the lumbar-pelvic-thigh complex can lead to inefficiencies or defects in other parts of the human body (Powers, 2003; Nadelr et al., 2000). In the upper part of the central stability zone, the most common injuries are seen in the neck-chest, spine-ribs, and shoulders (Mclean, 2002; Szeto et al., 2002; Hirashima et al., 2002; Bayes \& Wadsworth, 2009).

Chen et al. (1998) reported a sample of patients with scoliosis curvature in the thoracic lumbar region of 2267 degrees less balanced than healthy individuals. Beaulieu et al. (2009), said that patients with idiopathic scoliosis already have less balance and more fluctuations than their healthy peers. The severity of scoliosis may be related to the extent of sensory disturbances in patients.

The treatment of idiopathic scoliosis is considered practical Babris (Beaulieu et al., 2009). McIntire et al. (2008) recommend rotational strength training to be effective in reducing lateral spinal deviation and spinal stability in 20-40-degree scoliosis after 8 months and recommend spinal rotation training for scoliosis.

The use of traction to stabilize the spine has been considered in patients with scoliosis since Hippocrates. Pingot et al. (2007) examined and evaluated strength in children with idiopathic scoliosis. They suggested strength training for weak thigh and trunk muscles.
A review of the background literature and a review of research shows that the effects of corrective movements have been useful in correcting scoliosis (McIntire et al., 2008; Pingot et al., 2007). But in most of the previous measures, they have had only one or two types of exercises for the treatment of scoliosis, and with emphasis on strength or stretching exercises (Shakeri et al., 2018). However, in some studies, attention has been paid to the effect of spinal stability in the treatment of scoliosis (Najafi \& Seidi, 2014).

According to what has been explained above, the aim of the present study was to investigate whether there is a significant relationship between the application of central stability exercises with strength and stretching exercises for scoliosis-pathic. Will there be a significant difference in the extent of this association before and after the application of central stability exercises in the correction of scoliosis? Is there a significant relationship between stretching, strength training, and scoliosis abnormalities? Also, is there a significant relationship between stretching exercises, strength and scoliosis anomalies, and central stability?

\section{Methods}

The study was a quasi-experimental study. The statistical population of the study was 60 boy students with scoliosis aged 9 to 13 years. Due to moral and social restrictions in Iran, only boys samples were used. These participants were selected in a simple and accessible way from the correctional movement's center of Babol city of Mazandaran province and randomly divided into 3 equal groups: Experimental 1 (EG 1) with emphasis on stretching, strength training), Experiments 2 (EG 2) with emphasis on stretching and strength training and central stability and a control group (CG). The study was approved by the local ethics committee (Approval number: 8726/F.P, 18 November 2020, Department of Physical Education \& Sport Science, Mazandaran University of Science and Technology).

In order to enter the study, samples with structural scoliosis, the age range of 9 to 13 years, as well as written consent of the parents of the participants were required and should not have a history of trunk surgery or fractures and joint diseases of the spine, shoulder girdle, obvious malformation, Visible lower limb malformations, including genovolgum, genovarum, out-of-normal body weight, and the subject's unwillingness to continue the program, will be the conditions for excluding samples from this study. 
Subjects were asked to make corrective movements when entering the laboratory room to observe the necessary conditions of not having shoes, socks, and cover on the upper body to assess the spine. Information about the age, height, weight of the subjects should be asked to be in the desired location for evaluation. To evaluate scoliosis, a checkerboard was used. The test takers should be able to stand comfortably and without any contractions in the muscles so that the body weight is evenly distributed on the legs (Rajbi, 2001).

To better and more reliably assess the subjects with scoliosis behind the lattice plate, the true and apparent length of the lower limb was evaluated, as a short leg causes the pelvis to sag on the short leg, resulting in scoliosis. How to measure the length of the legs is as follows:

Actual length: From the upper anterior iliac spine to the inner ankle, the true length of the foot is obtained (Sokhangoi \& Afsharmand, 2020).

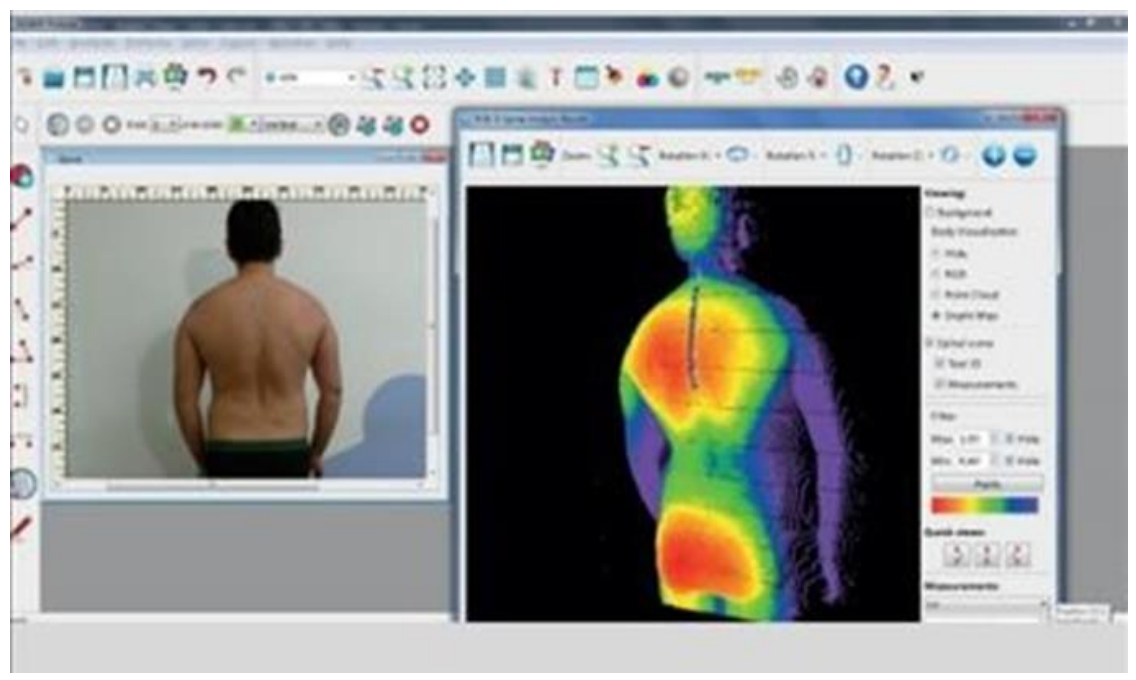

Figure 1. photographic measurement system.

Appearance length: They measure from the navel to the inner ankle and get the length of the appearance of the foot (Sokhangoi \& Afsharmand, 2020).

Participants with scoliosis whose true and apparent leg lengths are the same were measured for a complete evaluation of scoliosis angle balance using the photogrammetric method by marking the shock segments from c7 to s1 and with adobe pdf professional 10 software (Figure 1). In this simple field (2009), the validity of the mentioned method in measuring the scoliosis angle of the thoracic region compared to the radiographic report was high $(r=$
49\%) and stated that this method has a high reproducibility (ICC = 96\%) (Bergmark, 1989).

EG 1 performed the stretching and strength training, EG 2 carried out the stretching, strength, and central stability training and CG did not apply for any training or exercise program. During 8 weeks of training on EG 1 and EG 2, 3 training sessions for three weeks with a duration of 25 to 40 minutes per session under the direct supervision of two training groups participated in the training program.

CG tests were used in this modified exercise program of Park House et al. (2010) without performing any specific exercises and without knowing the status of other samples. At the beginning of each test session, the body was warmed up for 5 minutes, and then the members of the exercises group performed exercises in 6 stages with stretching, strength, and central stability exercises. In each session, the overload was applied incrementally and the repetition of the hands repetition increased the complexity of the movement and changed the level of reliance. Stretching exercises, strength-based exercises, and corrective movements that have been recommended in the book of corrective movements of Sokhangoi \& Afsharmand (2020) to correct the abnormality of postural scoliosis were performed in the training program for both exercises' groups. Finally, after eight weeks of the training program, all test subjects were evaluated again, and their scoliosis angle was measured by the photogrammetric method by marking on shock appendages from C7 to s1 vertebra (Rajabi \& Samadi, 2009); Information about the characteristics of the tests as well as the research changes in the two essays by descriptive. After completing the pre-test and post-test measurements in three groups, the Kolmogorov-Smirnov test showed that the distribution of the measured variables is normal and according to this, parametric tests were used. independent t-test to compare the information obtained between the exercises and control groups and paired t-test for Comparison of intragroup changes between pretest and posttest and also to determine the effectiveness of corrective exercises between the three groups of ANOVA were analyzed using SPSS 22.0 software. 


\section{Results}

The results showed that the mean spinal curvature angle of patients with scoliosis before and after participating in the correctional exercise program was EG 1, which showed a decrease of $2.97 \pm 0.17$ degrees, and the results of paired $t$-test showed that this reduction was statistically significant $(p=0.001)$. In EG 2 , which showed a decrease of $3.27 \pm 0.14$ degree and the results of paired t-test showed that this decrease was statistically significant $(p=0.001)$. Also, in the control group, it was $0.63 \pm 0.35$ degrees, which shows a decrease of 0.63 degrees, and the results of paired $t$ test showed that this decrease was not statistically significant $(\mathrm{p}=0.61)$.

According to the results of the independent $t$-test, although there was no significant difference between EG 1 and EG 2 in both pre-test and post-test, the test results showed that CG was significantly different from $\operatorname{EG} 1(p=0.001)$ and EG $2(p=0.001)$.

The effect size of corrective exercises in reducing scoliosis angle in EG 2 was larger than EG 1, which shows that combined corrective exercises in EG 2 were more effective than EG 1, but no significant difference was observed between EG 1 and EG 2 ( $p=0.63$ ).

The results also showed that there was a significant difference between the groups $\left(p=0.001 ; \mathrm{F}_{(58,2)}=8.38\right)$. CG was different from EG $1(p=0.003)$ and EG $2(p=$ $0.002)$ but there was no significant different between EG 1 and EG 2 ( $p=0.997)$.

\section{Discussion}

The aim of this study was to evaluate the effect of 8 weeks of stretching, strength, and central stability exercises on scoliosis in boy students aged 9 to 13 years. The results showed that the complication of scoliosis decreased in the subjects in the correctional exercise groups and there was a significant difference between the pre-test and post-test stages in EG 1 and EG 2 with the control group. These results were consistent with the findings of McIntire et al. (2008), Pingot et al. (2007), Naderi et al. (2019), Morningstar \& Joy (2006), and Gheitasi et al. (2014). Strength training affects the length of the lumbar muscle tendon and moves various skeletal parts, stabilizing the ligaments and making them stand.

\begin{tabular}{|c|c|c|c|c|}
\hline Variables & & Control $(n=20)$ & EG $1(n=20)$ & EG $2(n=20)$ \\
\hline Height $(\mathrm{cm})$ & & $141.4 \pm 7.8$ & $142.5 \pm 2.7$ & $142.4 \pm 9.6$ \\
\hline Weight (kg) & & $53.3 \pm 8.62$ & $52.4 \pm 7.31$ & $53.4 \pm 6.27$ \\
\hline Age (year) & & $10.2 \pm 89.1$ & $11.2 \pm 53.3$ & $11.2 \pm 30.7$ \\
\hline \multirow[t]{2}{*}{ LCA } & Pre-Test & $7.89 \pm 1.12$ & $8.81 \pm 1.67$ & $8.74 \pm 1.62$ \\
\hline & Post-Test & $7.26 \pm 1.47$ & $5.84 \pm 1.52$ & $5.47 \pm 1.48$ \\
\hline$p$-value & & 0.61 & $0.001 *$ & $0.001^{*}$ \\
\hline
\end{tabular}

Table 2

The one-way ANOVA for the effectiveness of exercises between the three groups.

\begin{tabular}{lcccccc}
\hline Source & SS & df & MS & $F$ & $p$ & Differences \\
\hline Between Groups & 104.57 & 2 & 52.28 & 8.38 & 0.001* & EG 1-CG \\
Within Groups & 262.0 & 58 & 6.23 & & & EG 2 - CG \\
Total & 366.57 & 60 & & & \\
\hline$* p<.05$ & & & & &
\end{tabular}


On the other hand, stretching exercises act as a coordinator of the pros and cons (Yakhdani et al., 2016). Ameri et al. (2008) concluded in their study that paraspinal disorders were one of the main causes of scoliosis. Therefore, it seems that strengthening the bilateral muscles of the spine through stretching and strength exercises can reduce one of the effective causes of scoliosis. So doing strengthening movements to weak muscles, flexible and stretching movements to shortened muscles, moving movements, and mobilizing movements to joints that have limited movement will be the most important cases.

The results show that in subjects with stretching, strength, and central stability exercises, exercises were more effective than subjects with stretching and strength exercises, which shows that combined exercises with emphasis on central stability can be more effective than exercises. With the emphasis on only strength and traction. Among the reasons for the better effectiveness of EG 2 can be trying to design exercises based on theoretical findings that cause abnormalities such as decreased irritability and muscular endurance in the spine, as well as reduced functional stability in the muscles of the central region and added squat exercises. Central was in addition to strengthening the weakened muscles and stretching the shortened muscles, as well as spinal irritability and improving the function of the trunk and central muscles of the body with emphasis on contraction and endurance to restore the correct direction of the spine.

Considering that the training programs have been performed in a suitable period ( 8 weeks) and direct and continuous supervision on the accuracy of individual exercises and the use of measurement methods with high reliability and reproducibility in scoliosis assessment, the control group has been used alongside the exercises group.

Based on the results of the present study, it is concluded that by designing principled and logical exercises based on updated research, combined and according to the needs of participants, age conditions and other issues in patients with scoliosis, including central stability exercises along with stretching and strength training. Corrective exercises are a safe and useful way to improve spinal abnormalities, especially scoliosis, and can reduce the problems and discomfort of participants with this complication.

Scoliosis is usually controlled with timely treatment. If any of the signs and symptoms of spinal deviation are observed by the physical education teachers of the country's schools, be sure to refer students to the basis of corrective movements and exercise therapy. Also, in order to reduce the progression of this complication and other complications of stature structure in students, to measure the stature structure of students at each academic level in a systematic way to identify these participants to prevent the progression of these complications in students and to treat patients.

\section{Financial support}

Financial support by Mazandaran University of Science and Technology (Project number: p/9048).

\section{Conflict of interest}

The authors declared no conflict of interest.

\section{References}

Ameri, E., Behtash, H., Mobini, B., Bouzari, B., Shoushtarizadeh, T., \& Kabiri, P. (2008). Investigation of pathology of paraspinal muscles in different scoliosis. Journal of Isfahan Medical School, 26(88), 46-53.

Balzini, L., Vannucchi, L., Benvenuti, F., Benucci, M., Monni, M., Cappozzo, A., Stanhope, (2003). Clinical characteristics of flexed posture in Elderly women. American Geriatrics Society, 51(10), 1419-1426.

Barrios, C., Tunon, M.T., Engstrom, W., \& Canadell, J. (1989). Paraspinal muscle Pathology in experimental scoliosis. Arch other Trauma Surg, 108(6), 342-345.

Bayes, M.C., \& Wadsworth, L.T. (2009). Upper extremity injuries in golf. Phys Sports Med, 37(1), 92-96.

Beaulieu, M., Toulotte, C., Gatto, L., Rivard, C. H., Teasdale, N., Simoneau, M., \& Allard, P. (2009). Postural imbalance in non-treated adolescent idiopathic scoliosis at different periods of progression. Eur Spine J, 18(1), 38-44.

Behtash, H., Ganjavian, M., Shahre Babaki, B., Fereshtehnejad, S.M, \& Akbarnia, B. (2006). The effect of halo traction between anterior and posterior spinal fusion on the correction of scoliosis. Tehran Univ Med J, 64(10), 6-14.

Bergmark, A. (1989). Stability of the lumbar spine. A study in mechanical engineering. Acta Other Scand, 230(suppl), 20-44.

Chen, P. Q., Wang, J. L., Tsuang, Y. H., Liao, T. L., Huang, P. I., \& Hang, Y. S. (1998). The postural stability control and gait pattern of idiopathic scoliosis adolescents. Clinical Biomechanics, 13(1), S52-S58. 
Crisco, J.J., \& Panjabi, M.M. (1991). The intersegmental and multisegmental muscles of the spine: A biomechanical model comparing lateral stabilizing potential. Spine, 7, 793-799.

Forghani Ozrudi, M.B., \& Nikkhoo Amiri, F. (2021). Evaluation of Kyphosis in Mazandaran University of Science and Technology students and its relation to psychological factors. Turk J Kinesiol, 7(1), 39-43.

Gheitasi, M., Alizadeh, M.H., Rajabi, R., Ebrahimi Takamjani, I., \& Haghverdian, S. (2014). The effect of 8 weeks of regular corrective exercises (stretching-strengthening exercises) on the amount of lateral curvature of the spine in participants with unstructured scoliosis using photogrammetry. J Appl Sport Psychol, 10(20), 93-106.

Ghiasi, F., Akbari, A., \& Sangtarash, F. (2007). The effect of stabilization method and Williams exercise on improvement of functions in patients with mechanical chronic low back pain. J Shahrekord Univ Med Sci, 8(4), 21-28.

Hirashima, M., Kadota, H., Sakurai, S., Kudo, K., \& Ohtsuki, T. (2002). Sequentail muscle activity and its functional role in the upper extremity and trunk during overam throwing. J Sports Sci, 20, 301-310.

Mahaudens, P., Thonnard, J.L., Detrembleur, C. (2005). Influence of structural pelvic disorders during standing and walking in adolescents with idiopathic scoliosis. Spine J, 5(4), 427-433.

McIntire, K. L., Asher, M. A., Burton, D. C., \& Liu, W. (2008). Treatment of adolescent idiopathic scoliosis with quantified trunk rotational strength training: a pilot study. Clin Spine Surg, 21(5), 349-358.

Mclean, L. (2002). The effect of postural correction on muscle activation amplitudes recorded form the cervicobrachial region. J Electromyogr Kinesiol, 15, 527-535.

Morningstar, M.W., \& Joy, T. (2006). Scoliosis treatment using spinal manipulation and the Pettibon Weighting System a summary of 3 atypical presentations. Chiropr Man Therap, 14, 1. https://doi.org/10.1186/1746-134014-1

Nadler, S. F., Malanga, G. A., DePrince, M., Stitik, T. P., \& Feinberg, J. H. (2000). The relationship between lower extremity injury, low back pain, and hip muscle strength in male and female collegiate athletes. Clin J Sport Med, $10(2), 89-97$.

Naderi, A., Rezvani, M.H., Shaabani, F., \& Bagheri, S. (2019). Effect of Kyphosis exercises on physical function, postural control and quality of life in elderly men with hypokyphosis. Salmand: Iranian Journal of Ageing, 13(4), 464-479.

Najafi, H., \& Seidi, F. (2014). The effect of an eight-week selected corrective exercises program on non-structural scoliosis deformity. Journal for Research in Sport Rehabilitation, 2(3), 11-19.

Naqvi, U. (2019). Muscle strength grading. FL: StatPearls Publishing.

Pingot, M., Czernicki, J., \& Kubacki, J. (2007). Assessment of muscle strength of hip joints in children with idiopathic scoliosis. Ortopedia, Traumatologia, Rehabilitacja, 9(6), 636-643.

Powers, C.M. (2003). The influence of altered lowerextremity kinematic on patellofemoral joint dysfunction: a theoretical perespectiv. J Orthop Sports Phys Ther, 33(11), 639-646.

Rajbi, R. (2001). The inverstigation and comparison of scoliosis in special and common in students 11-15 School. J Sci Physiotr, 21(2), 56-58.

Rajabi, R., \& Samadi, H. (2009). Guide to the laboratory of corrective movements. Tehran: University of Tehran Publishing Institute.

Richardson, C.A., \& Jull, G.A. (1995). Muscle control-pain control. What exercises would you prescribe? Man Ther, 1, 2-10.

Richarson, C.A., Jull, G. Toppenberg, R., \& Comerford, M. (1992). Techniques for active lumbar stabilization for spinal protection:a pilot study. Aust J Physiother, 38, 105112.

Sadd, R.K., Colombo, A.S., \& Amado Joao S.M. (2009). Reliability and Validity of the photogrammetry for scoliosis evaluation: a cross-sectional prospective study. Journal of Manipulative and Physiological Therapeutics, 32(6), 423-430.

Schmidt, R.A., \& Lee, T.D. (2011). Motor Control and learning: a behavioral emphasis. 5th ed. Champaign, IL: Human Kinetics.

Shakeri, A., Sokhangoie, Y., \& Hoseini, Y. (2018). The effects of a course of corrective exercises on postural and biomechanical parameters in patients with idiopathic scoliosis by using photogrammetry method. HRJBAQ, $3(2), 73-81$.

Sokhangoi, Y., \& Afsharmand, Z. (2020). Corrective movements. Tehran: Publication of Hatami Publications and Partners.

Szeto, G.P.Y., Straker, L., \& Rain, S. (2002). A field comparison of neck and shoulder postures in symptomatic and asymptomatic office workers. Appl Ergo, 33, 57-84.

Tse, M. A., McManus, A. M., \& Masters, R. S. (2005). Development and validation of a core endurance intervention program: implications for performance in college-age rowers. J Strength Cond Res, 19(3), 547-552.

Yakhdani, H. F., Kamran, S., Abasi, H., Kordi, M. H., \& Abedinzadeh, S. (2016). Comparison of eight weeks of 
training in the water and land on changes in lumbar lordosis of girl students of Yazd City. J Clin Physiother Res, 2(4), 182-186.
Zazulak, B.T., Hewett, T.E., Reeves, N.P., Goldberg, B., \& Cholewicki, J. (2007). Deficits in neuromuscular control of the trunk predict knee injury risk. Am J Sports Med, 35(7), 1123-1130. 